\title{
Remote Postconditioning Cardio-protection After Myocardial Ischemia/reperfusion Injury by Eliminating 4-HNE and Regulating Autophagy Through ALDH2/SIRT3/HIF1a Signaling Pathway
}

\section{Rifeng Gao}

Fifth People's Hospital of Shanghai Fudan University https://orcid.org/0000-0002-3315-1847

Chunyu Lv

Peking University Shenzhen Hospital

Yanan Qu

Zhongshan Hospital Fudan University

Hen Yang

Nanchang University Second Affiliated Hospital

Chuangze Hao

Nanchang University Second Affiliated Hospital

\section{Xiaolei Sun}

Zhongshan Hospital Fudan University

\section{Xiaosheng Hu}

First Hospital of Zhejiang Province: Zhejiang University School of Medicine First Affiliated Hospital

\section{Yiqing Yang}

Fifth People's Hospital of Shanghai Fudan University

\section{Yanhua Tang ( $\nabla$ tyh6565@163.com )}

Nanchang University Second Affiliated Hospital https://orcid.org/0000-0002-8563-8323

\section{Research Article}

Keywords: Remote postconditioning, Ischemia/reperfusion injury, Aldehyde dehydrogenase 2, HIF1a, SIRT3, 4-HNE

Posted Date: February 23rd, 2022

DOI: https://doi.org/10.21203/rs.3.rs-1301675/v1

License: (c) (1) This work is licensed under a Creative Commons Attribution 4.0 International License. Read Full License 


\section{Abstract \\ Purpose}

Remote postconditioning (Rpost) was proposed based on postconditioning studies. Aldehyde dehydrogenase 2 (ALDH2) is a unique endogenous cardioprotective enzyme in the mitochondria, associated with various cardiovascular diseases. However, whether Rpost exerts myocardial protection through ALDH2 has not been systematically reported.

\section{Methods}

C57BL/ 6 and ALDH2 knockout (ALDH2-KO) mice were subjected to left anterior descending ligation to construct ischemic/reperfusion (I/R) models. Before the start of reperfusion after myocardial ischemia in mice, the bilateral hindlimbs were used as target organs to undergo Rpost.

\section{Results}

Echocardiography and single-cell contraction experiments showed that Rpost significantly improved myocardial function and alleviated myocardial ischemia-reperfusion injury in C57BL/6 mice after l/R, but it was challenging to exert myocardial protection in ALDH2-KO mice. TUNEL, Evans blue/TTC, and reactive oxygen species (ROS) assay results showed that the effect of Rpost on reducing myocardial cell apoptosis, myocardial infarction area, and myocardial ROS levels was not significantly different in the $\mathrm{MI} / \mathrm{RI}$ in ALDH2-KO mice. Electron microscopy and western blotting showed that Rpost could upregulate ALDH2 expression, activate sirtuin 3 (SIRT3)/hypoxia-inducible factor 1-alpha (HIF1a), regulate autophagy, and exert myocardial protection. However, this protective effect may be blocked in the absence of ALDH2. Furthermore, we found that Rpost can upregulate the expression of ALDH2 and inhibit the production of 4-hydroxynonenal (4-HNE), playing a protective role in the myocardium.

\section{Conclusion}

This study revealed that Rpost could exert myocardial protection through the ALDH2/SIRT3/HIF1a signaling pathway by reducing 4-HNE secretion. The study explores the feasibility of Rpost in ALDH2deficient populations, thereby providing a basis for the targeted clinical application of Rpost.

\section{Highlights}

- Rpost protects myocardium from I/R injury by upregulating ALDH2 expression.

- Rpost cardio-protection regulates autophagy through ALDH2/SIRT3/HIF1a signaling pathway. 
- The study explores the feasibility of Rpost in ALDH2-deficient populations, thereby providing a basis for the targeted clinical application of Rpost.

\section{Introduction}

Myocardial ischemia/reperfusion injury (MI/RI) is common in the clinical treatment of cardiovascular diseases[1]. For example, reducing MI/RI after opening the aorta during cardiopulmonary bypass surgery and coronary stent implantation in coronary heart disease has consistently been the focus of general cardiovascular clinical attention[2]. Preconditioning and proximal postconditioning can play a role in myocardial protection; however, they have low applicability and are difficult to promote due to the difficulty in operation [3-5]. Remote postconditioning (Rpost) is a new concept proposed based on postconditioning, which refers to the short-term $\mathrm{l} / \mathrm{R}$ of another organ before reperfusion after ischemia to activate the endogenous protective mechanism of the body to play a role. However, the specific mechanism of action of Rpost remains unclear. Therefore, exploring the mechanism of Rpost can provide a basis for the clinical application of Rpost[5, 6].

Aldehyde dehydrogenase 2 (ALDH2), an essential isoenzyme in the ALDH family, is an endogenous cardioprotective factor in the mitochondria closely related to cardiovascular disease occurrence and is involved in the pathology of coronary heart disease, heart failure, cardiomyopathy, and several other physiological processes $[7,8]$. It is distributed in various tissues and organs of the human body, mainly in the mitochondria of the human heart, brain, lung, liver, and kidney cells[8, 9]. The level of ALDH2 in the heart is much higher than that of other types of aldehyde dehydrogenases; and it has the most robust activity. Approximately $40 \%$ of the East Asian population has the ALDH2 deletion genotype closely related to myocardial infarction (MI)[10]. Furthermore, recent studies have shown that a variety of preconditioning and postconditioning strategies can play a role in MI/RI through ALDH2, indicating that ALDH2 is crucial for pre -and post-stimulation[9, 11]. In addition, some studies suggest that ALDH2 may block the protective effect of Rpost, but its specific mechanism remains unclear[12, 13].

In conclusion, Rpost can reduce Ml/Rl; however, its research is still at the experimental level, and there are numerous challenges to be examined. In addition, there is no apparent report on the role of ALDH2 in the protection of the Rpost myocardium. Therefore, we hypothesized that Rpost could activate endogenous ALDH2 to protect the myocardium. In this study, ALDH2 knockout (ALDH2-KO) and wild-type mice were used to establish I/R models and perform Rpost treatment to observe the role of ALDH2 in Rpost-induced myocardial protection and related mechanisms.

\section{Materials And Methods}

\subsection{Animals}

Wild-type mice (C57BL/6 age 8-10 weeks) were obtained from Cavens Biogle Model Animal Research Co., Ltd. (Suzhou, China), and matched ALDH2-KO male mice were generated as described previously[14]. All 
animal studies were performed in accordance with the guidelines evaluated and approved by the Animal Ethics Committee of Fudan University. Experimental animals were carried out in laboratory Animal Center of Zhongshan Hospital affiliated to Fudan University.

\subsection{MI/RI and Rpost treatment}

To establish a mouse MI/RI model, we used 8-10 weeks mice. Anesthesia was maintained under $2 \%$ isoflurane induction (RWD Life Science Inc., Shenzhen, China). The limbs of mice were fixed, the left chest of the mice was exposed, and hair was shaved. We selected four or five intercostal openings on the left side of the mouse, separated the muscles, identified the strongest point of the apex, inserted the mosquito vascular clamp, opened the chest, and applied slight pressure on the right index finger to make the mouse heart jump out of the chest cavity. The mouse left ventricular descending anterior descending branch was ligated with a 6-0 silk suture slipknot. After ligation, the mouse heart was returned to the heart cavity, and a thread with two slip knots was placed outside the chest cavity. The wound was sutured, and the knot was loosened after waiting for 45 minutes. The detailed method is explained previously[15]. During this period, a $3 \times 5$ tourniquet latex tube was used to ligate mouse legs to block the blood flow for 5 min and then released for 5 min three times for Rpost treatment.

\subsection{Echocardiography analysis}

Mice were anesthetized with isoflurane to evaluate cardiac function after MI/RI, and M-mode images were acquired using a Vevo 2100 high-frequency ultrasound system (VisualSonics, Toronto, ON, Canada). The data were averaged based on the measurements of at least six cardiac cycles, including recording left ventricular ejection fraction (LVEF) and left ventricular fractional shortening (LVFS) scores. The specific operation was performed as described previously[15, 16].

\subsection{Single-cell systolic and diastolic function of cardiomyocytes}

Cardiomyocytes were isolated from the model mice using a previously described method ${ }^{[17]}$. The systolic and diastolic functions of primary cardiomyocytes were detected using the lonOptix ${ }^{\mathrm{TM}}$ system. The detection buffer, cardiomyocyte calcium buffer, comprised $130 \mathrm{mM} \mathrm{NaCl}, 5.4 \mathrm{mM} \mathrm{KCl}, 10 \mathrm{mM}$ HEPES, 1.8 $\mathrm{mM} \mathrm{CaCl}_{2}, 0.5 \mathrm{mM} \mathrm{MgCl}_{2}$, and $10 \mathrm{mM}$ glucose, $\mathrm{pH}$ 7.4. Two drops of buffer were added to each slide. The contractile function of cardiomyocytes was evaluated by measuring the peak shortening (PS) and maximal velocity of shortening (-dL/dt) of cardiomyocytes.

\subsection{Evan's blue/triphenyltetrazolium chloride (TTC) staining}

After $24 \mathrm{~h}$ of reperfusion, the mice were anesthetized intraperitoneally with $2 \%$ sodium pentobarbital. The chest cavity was opened, the heart was exposed, the anterior coronary artery was retied, and $1 \%$ Evans blue was injected into the left atrial appendage to allow the heart to beat freely. The heart was then cut out, washed with PBS, and immediately frozen on dry ice. After $30 \mathrm{~min}$, the heart was cut into 5-6 shortaxis sections on an average with a blade. Next, $1 \%$ TTC was incubated at $37^{\circ} \mathrm{C}$ in a water bath for 30 min. Each section was flattened and fixed with $4 \%$ paraformaldehyde for $2 \mathrm{~h}$. The blue area impregnated with 
Evans blue was the non-ischemic area. The red area impregnated by TTC was the ischemic area. The white unstained area was the myocardial infarction site. The area at risk (AAR) included both ischemic and myocardial infarction areas. Image quantification was performed by segmenting the stained areas of each section using ImageJ software, as described previously[15]. The infarction area is expressed as the percentage of AAR generated.

\subsection{TUNEL assay}

Myocardial tissues (6-8 mice per group, two cuts at the myocardial AAR) were fixed with $4 \%$ paraformaldehyde and stained using the One Step TUNEL Apoptosis Assay Kit (Beyotime Biotechnology, China) according to previous reports[15].

\subsection{Reactive oxygen species (ROS) measurement}

ROS production was evaluated by analyzing the fluorescence intensity resulting from dihydroethidium (DHE) staining (Invitrogen D11347). Briefly, frozen mice hearts were cut into $5 \mu \mathrm{m}$ sections. The heart sections were stained 37 times with $5 \mu \mathrm{M}$ DHE for 30 min followed by staining with DAPI for 10 min and observed by using fluorescence microscope.

\subsection{Histological analysis}

The myocardial tissue was fixed with 4\% paraformaldehyde (6-8 mouse in each group, two incisions at the connective tissue of myocardial infarction) 24 hours after reperfusion. Macrophage infiltration was detected using anti-mouse F480 (ab25377; Abcam). The detailed method is described previously[16].

\subsection{Electron microscopy}

Transmission electron microscopy was used to observe the ultrastructure of the cardiomyocytes. Briefly, the hearts of mice in each experimental group were perfused and fixed with tube-buffered formaldehydeglutaraldehyde. The left ventricular myocardium was removed from the middle of the ventricle and cut into $1-\mathrm{mm}^{3}$ pieces. The blocks were fixed with a 10:1 liquid/tissue ratio at $4^{\circ} \mathrm{C}$ overnight. To further process the myocardial mass, it was incubated in $2 \%$ sucrose $(\mathrm{pH} 7.4), 1 \% \mathrm{OsO}_{4}$, and $1.5 \%$

$\mathrm{K}_{3}\left[\mathrm{Fe}(\mathrm{CN})_{6}\right] \cdot 3 \mathrm{H}_{2} \mathrm{O}$ buffer overnight at $22-24^{\circ} \mathrm{C}$. It was then dehydrated with graded ethanol and propylene oxide and finally encapsulated with Epon/Araldite. An RMC-MTXL ultramicrotome and diatom diamond knife were used to obtain sections. The images were obtained using a CM-120 transmission electron microscope (Philips, Netherlands). Each mouse heart sample was observed in at least 10 fields.

\subsection{Western blot analysis}

Cardiac tissue at the infarct site was harvested in the RIPA lysis buffer containing $1 \mathrm{mM}$ phenylmethanesulfonyl fluoride. Protein samples were separated by $10 \%$ and $15 \%$ SDS-PAGE and transferred to polyvinylidene difluoride membranes (Biotech Well). The membranes were blocked with $5 \%$ bovine serum albumin in TBST for $2 \mathrm{~h}$ and incubated with the following primary antibodies at $4^{\circ} \mathrm{C}$ overnight: ALDH2 (ab133306; Abcam; 1:1000), hypoxia-inducible factor 1-alpha (HIF1a) (36169S; Cell Signaling Technology; 1:1000), 4-hydroxynonenal (4-HNE) (ab46545, Abcam; 1:3000), sirtuin 3 (5490S; 
Cell Signaling Technology; 1:1000), P62 (ab109012; Abcam; 1:10000), LC3B (ab192890; Abcam; 1:2000), caspase-3 (19245S; Cell Signaling Technology; 1:1000), cleaved caspase-3 (9664S; Cell Signaling Technology; 1:1000), Bax (14796S; Cell Signaling Technology; 1:1000), BCL-2 (3498S; Cell Signaling Technology; 1:1000), and $\beta$-actin (4970S; Cell Signaling Technology; 1:4000). The horseradish peroxidase-conjugated secondary antibody was allowed to stand at room temperature $\left(24^{\circ} \mathrm{C}\right)$ for 1.5 hours. Detection was performed using Immobilon Western chemiluminescent HRP substrate (Millipore, Billerica, MA, USA). Gel images were captured using an Image Quant LAS 4000 Mini (GE Healthcare, Barrington, IL, USA).

\subsection{Statistical analysis}

Data are expressed as the mean \pm standard error of the mean (SEM). Statistical analyses were conducted using GraphPad Prism 5.01 software. The normality of the data distribution was tested using the Kolmogorov-Smirnov test. The Mann-Whitney-U test was used when the group data were not normally distributed or if the group variances were unequal. The homogeneity of variance test was performed using Levene's test. Continuous data with normal distribution were assessed by one-way ANOVA with post hoc test or two-way ANOVA with post hoc test (Tukey-Kramer) as indicated[16].

\section{Results}

\subsection{Rpost treatment preserves cardiac function after I/R}

To investigate the therapeutic potential of Rpost in vivo, we used an I/R mouse model. I/R injury was mimicked by coronary artery ligation for $45 \mathrm{~min}$, followed by $24 \mathrm{~h}$ reperfusion with Rpost. Figure $1 \mathrm{~A}$ shows representative echocardiograms illustrating the comparison of mouse hearts after surgery and those in the sham group $24 \mathrm{~h}$ after treatment with Rpost. The heart rate of the mice was similar in all groups (Figure 1B). In the sham groups, it was evident that Rpost treatment did not change the LVEF (Figure 1C) or LVFS (Figure 1D). After $24 \mathrm{~h}$ of reperfusion, echocardiographic parameters were significantly restored in mice treated with Rpost compared with those in the control group; the LVEF values were: $\mathrm{l} / \mathrm{R}+\mathrm{Rpost}$ group $(61.89 \pm 4.120 \%, \mathrm{~N}=10)$ and $\mathrm{l} / \mathrm{R}$ group $(49.77 \pm 3.399 \%, \mathrm{~N}=10)$, with $\mathrm{p}$ values $<0.05$ (Figure 1C) and the LVFS values were I/ $R+R$ post group $(34.73 \pm 1.952 \%, N=10)$ and $I / R$ group $(21.00 \pm 2.638 \%, N=10)$, with $p<0.001$ (Figure 1D).

After isolating the mouse cardiomyocytes in each group, we used the lonOptix ${ }^{\mathrm{TM}}$ Soft-Edge single-cell contractile function detection system to evaluate the functional improvement of Rpost in mouse cardiomyocytes after I/R at the cellular level. In terms of systolic function, PS and -dL/dt in failed cardiomyocytes after I/R were significantly lower than those in the sham group, and they were significantly improved after Rpost treatment (Figure 1E and 1F). Furthermore, single-cell contractile function analyses demonstrated that Rpost could increase PS and -dL/dt (Figure 1E and 1F) after I/R.

\subsection{Rpost treatment ameliorated I/R injury in mice}


To further explore the therapeutic effect of Rpost after I/R, we assessed the myocardial infarction area and myocardial apoptosis in mice. Myocardial infarction size was assessed by Evans blue dye and TTC staining, and a smaller infarction size was observed after Rpost treatment, as shown by a reduced ratio of the white-to-red area (Figure 2A-C). Furthermore, the results of apoptotic protein analysis showed that after I/R, the expression of myocardial apoptotic protein Bax and cleaved caspase-3 increased significantly (Figure 2D, 2E, and 2G). Conversely, after Rpost treatment, the expression of Bax and cleaved caspase-3 were significantly decreased (Figure 2D, 2E, and 2G). TUNEL staining results showed that Rpost significantly reduced cardiomyocyte apoptosis (Figure $2 \mathrm{~F}$ and $2 \mathrm{H}$ ). In addition, we examined the ROS deposition levels in the frozen sections using DHE staining. Rpost treatment significantly reduced postoperative injury after I/R (Figure 3A and $3 C$ ). F480 results also showed that Rpost significantly reduced macrophage infiltration in myocardial tissues (Figure 3B and 3D).

\subsection{Rpost protects myocardium from I/R injury through ALDH2/SIRT3-based regulation of autophagy and reduction of 4-HNE levels in mice}

ALDH2 is an endogenous protective factor that plays an essential role in repairing $1 / R$ injury[10]. Our previous research also showed that Alda- 1 treatment promotes the therapeutic effect of mitochondrial transplantation in MI/RI[15]. In our protein experiment, we found that after I/R, the expression of ALDH2 was significantly downregulated, but Rpost treatment significantly promoted the expression of ALDH2 (Figure 3E and 3F). The expression of SIRT3 was similar to the expression of ALDH2, which was downregulated after I/R, and up-regulated after Rpost the change was also obvious (Figure 3E and 3F). In addition, Rpost decreased myocardial autophagy after I/R (Figure 3E and 3G). The electron microscopy results showed that the arrangement of the myocardium was disordered, and the number of autophagosomes increased after I/R (Figure 3H). Post-treatment, the arrangement of the myocardium was improved, and the number of myocardial autophagosomes was reduced. We also found that Rpost attenuated 4-HNE induction by MI/RI, further reducing ROS levels and myocardial apoptosis (Figure 3I). Hence, we speculate that Rpost protects the myocardium from I/R injury through autophagy by regulating the ALDH2/SIRT3 signaling pathway and through attenuation of 4-HNE by the ALDH2/4-HNE signaling pathway in mice.

\subsection{ALDH2 deficiency blocks protective effect of Rpost on I/R injury in mice}

To explore the relationship between ALDH2 and Rpost, we selected ALDH2-KO mice to construct an I/R model to explore the mechanism of Rpost further. After $24 \mathrm{~h}$ of reperfusion, echocardiography examination revealed worse LV functions in ALDH2-KO mice than in the WT controls, presenting lower EF and $\mathrm{FS}$ values in ALDH2KO mice (Figure 4A, 4C, and 4D). Furthermore, we used the lonOptix ${ }^{\mathrm{TM}}$ Soft-Edge single-cell contractile function detection system to evaluate cardiac function. The study found that PS (Figure 4E) and -dL/dt (Figure 4F) in the ALDH2-KO group were worse than those in the WT group. In 
addition, Rpost significantly improved I/R-induced myocardial dysfunction in the WT group but not in the ALKDH-KO group (Figure 4).

Evan's blue/TTC staining and TUNEL staining showed that in both the control and Rpost groups, the area of myocardial infarction and myocardial apoptosis in the ALDH2-KO group increased significantly (Figure 5). The results of apoptotic protein further proved that the myocardial protective effect of Rpost could be blocked by ALDH2 (Figure 6A-E). In addition, ROS and F480 staining showed that Rpost could reduce ROS levels and myocardial inflammation after I/R in wild-type mice, but the therapeutic effect was blocked in the ALDH2-KO group (Figure 6F-I). Furthermore, ROS and myocardial inflammation in the ALDH2-KO group increased significantly after I/R compared with that in the WT group (Figure 6F-I).

The results of the appeal study show that ALDH2 is crucial for the action of Rpost. After Rpost treatment, the area of myocardial infarction in the WT group was reduced and myocardial apoptosis and ROS levels were significantly decreased, but there was no significant improvement in the ALDH2-KO group (Figure 5 and 6). In summary, the above results show that Rpost can protect mouse myocardium from I/R injury, but ALDH2 can block this protective effect.

\subsection{Rpost exhibits cardio-protection after MI/RI by eliminating 4-HNE and regulating autophagy through ALDH2/SIRT3/HIF1a signaling pathway}

Rpost can cause transient ischemia in the lower limbs of mice, induce high expression of HIF1a, and exert myocardial protection (Figure 7A and 7C). However, in the ALDH2-KO group, Rpost did not induce high expression of HIF1 a (Figure 7A and 7C). SIRT3 is one of several nicotinamide adenine dinucleotidedependent histone deacetylases that regulate various functions in mammals, including aging and metabolism[18, 19]. ALDH2 is a direct SIRT3 substrate, and its deacetylation increases acetaminophen toxic-metabolite binding and enzyme inactivation[20]. Under normal circumstances, the deletion of ALDH2 did not affect the expression of SIRT3 (Figure 7A and 7D). However, after I/R in the WT group, the expression of SIRT3 was significantly reduced, and Rpost treatment significantly promoted the expression of SIRT3 (Figure 7A and 7D). However, when ALDH2 was deficient, SIRT3 expression in the I/R group was significantly decreased, but Rpost did not increase SIRT3 expression (Figure 7A and 7D). In addition, we found that myocardial autophagy was significantly increased after $\mathrm{I} / \mathrm{R}$, and Rpost inhibited myocardial autophagy (Figure 7A, 7E, and 7F). However, when ALDH2 was deficient, the regulatory effect of Rpost on autophagy disappeared (Figure 7A, 7E, and 7F). Furthermore, Rpost can reduce the induction of 4-HNE after MI/RI, but the effect of Rpost was blocked by ALDH2 deletion (Figure 7G). In summary, we speculate that Rpost protects the myocardium from I/R injury through autophagy regulated by the ALDH2/SIRT3/HIF1a signaling pathway and attenuates 4-HNE via the ALDH2/4-HNE signaling pathway in mice (Figure $7 \mathrm{H})$.

\section{Discussion}


To our understanding, this is the first study to demonstrate that Rpost can regulate autophagy through the ALDH2/SIRT3/HIF1a signaling pathway and attenuates 4-HNE protecting the myocardium from I/R injury. Rpost is feasible and straightforward and is expected to be a more promising myocardial protection measure in clinical applications. Using this strategy, we observed a significant improvement in myocardial function after I/R injury in mice. In addition, our study demonstrated that Rpost could attenuate 4-HNE and regulate myocardial autophagy through the ALDH2/SIRT3/HIF1a pathway, reduce myocardial infarction area, and inhibit myocardial cell apoptosis. This study suggests that Rpost plays a vital role in cardiac protection after I/R injury.

Myocardial ischemia-reperfusion injury is widely used in the clinical treatment of cardiovascular diseases, such as aortic opening after cardiopulmonary bypass surgery and coronary artery stent implantation. Rpost can initiate the endogenous protective mechanism of the body by temporary ischemia-reperfusion of another organ before the onset of myocardial ischemia-reperfusion. Previous studies have shown that Rpost can reduce infarct size, protect myocardial function, and improve adverse cardiac remodeling in patients with MI[21, 22]. Furthermore, Rpost can improve the inflammatory response after cerebral ischemia and reduce cerebral hemorrhage and the risk of cerebral hemorrhage [23]. As an exogenous intervention, Rpost is simple and easy to implement. It can improve the clinical prognosis of patients with myocardial infarction in various ways, but the specific mechanism of Rpost remains unclear. In this study, a mouse I/R model was constructed, and post-treatment was performed. It was found that Rpost can significantly reduce the area of myocardial infarction and myocardial cell apoptosis and protect heart function. However, when ALDH2 is deficient, the myocardial protection of Rpost disappears. In addition, we found that Rpost significantly promoted the expression of ALDH2 in the myocardium.

ALDH2 is an endogenous cardioprotective factor in the mitochondria and is involved in the pathophysiological processes of coronary heart disease, heart failure, cardiomyopathy, and several other diseases [24-26]. Ma et al. performed ischemia-reperfusion treatment in wild mice, ALDH2-overexpressed mice, and ALDH2-KO mice and found that the area of myocardial infarction in ALDH2-overexpressed mice was significantly decreased, whereas that in ALDH2-KO mice was increased [27]. Our research shows that after I/R, ALDH2 expression was significantly decreased, and Rpost can significantly increase the expression of ALDH2. Furthermore, we found that Rpost upregulated the expression of ALDH2 and SIRT3. SIRT3 is mainly located in the mitochondria and can reduce oxidative stress damage and the area of myocardial infarction by activating the anti-oxidative stress signaling pathway, thereby protecting the myocardium from reperfusion injury [28]. ALDH2 is a direct SIRT3 substrate, and its deacetylation increases acetaminophen toxic-metabolite binding and enzyme inactivation[20,29]. Therefore, we believe that Rpost can reduce myocardial ischemia-reperfusion injury by promoting the expression of ALDH2/SIRT3, thereby exerting myocardial protection.

Myocardial ischemia-reperfusion injury stabilizes the transcription factor HIF1a, the primary regulator of the transcriptional response initiated by hypoxia ${ }^{[31]}$, and HIF2a[30]. ALDH2 can regulate mitochondrial fission and smooth muscle cell proliferation via the 4-HNE/HIF1a signal pathway[31, 32]. Thus, ALDH2 acts as an endogenous cardiac protective factor in the mitochondria and can exert myocardial protection 
by regulating autophagy [33]. The results of this study prove that Rpost can regulate autophagy through the ALDH2/SIRT3/HIF1 a pathway to exert myocardial protection, reduce myocardial ischemia-reperfusion injury and myocardial infarction area, inhibit myocardial cell apoptosis, and improve cardiac function,. In addition, we found that Rpost reduced myocardial inflammation after I/R.

Mitochondria are the main source of ROS in cells, and when ROS exceed their antioxidant capacity, they lead to fatty acid oxidation, a process known as lipid peroxidation[34]. 4-HNE is the most abundant lipid peroxidation product and forms adducts with proteins, which affects its biological function and destroys intracellular homeostasis[35]. The level of plasma 4-HNE was increased in patients with HF, which was negatively correlated with cardiac function[36]. ALDH2 is a mitochondrial enzyme that metabolizes ethanol and toxic aldehydes, such as 4-HNE[37]. In this study, we found for the first time that MI/RI leads to excessive 4-HNE levels in the mitochondria and has serious consequences in cardiac dysfunction after I/R. Conversely, Rpost can upregulate ALDH2 expression, clear excessive 4-HNE levels, protect the myocardium, and reduce ROS levels.

Notably, $40 \%$ of the East Asian population and $8 \%$ of the global population carry the ALDH2 mutation, which is caused by the replacement of glutamate with lysine at amino acid 487 and results in only $15 \%$ of the catalytic activity of the wild-type ALDH2[38-40]. Our present study indicates that Rpost can protect the myocardium from I/R damage through the ALDH2/SIRT3/HIF1 a pathway and by decreasing 4-HNE levels. Furthermore, Rpost is highly operable, simple, easy to implement, and significant for clinical transformation. Therefore, it is expected to become a myocardial protection measure with more clinical application prospects.

\section{Abbreviations}

Rpost: Remote postconditioning; ALDH2: Aldehyde dehydrogenase 2; I/R : ischemic/reperfusion; ROS: reactive oxygen species; MI/RI: Myocardial ischemia/reperfusion injury; ALDH2-KO: ALDH2 knockout; SIRT3: sirtuin 3; HIF1 a: hypoxia-inducible factor 1-alpha; 4-HNE: 4-hydroxynonenal; DHE: dihydroethidium; MI: myocardial infarction; LVEF: left ventricular ejection fraction; LVFS: left ventricular fractional shortening; TTC: triphenyltetrazolium chloride; AAR: area at risk; WT: wild-type.

\section{Declarations}

\section{Data availability statement}

The data that support the findings of this study are available from the corresponding author upon reasonable request.

\section{Acknowledgments}

None 


\section{Funding}

This research was funded by the Jiangxi Provincial Natural Science Foundation Project (20071BBG70067). This work was supported by the National Natural Science Foundation of China (\#81160019 and \#81360031).

\section{Author contributions}

Rifeng Gao, Chunyu Lv and Yanan Qu designed the research and wrote the paper. Rifeng Gao, Heng Yang, Xiaolei Sun and Chuangze Hao performed the experiments. Rifeng Gao, Xiaosheng Hu, Yiqing Yang and Yanhua Tang contributed new reagents/analytic tools and provide critical suggestions. Rifeng Gao, Chunyu Lv and Yanan Qu analyzed the data. Xiaosheng Hu, Yiqing Yang and Yanhua Tang edited the paper.

\section{Conflict of interest statement}

The authors declare that there is no conflict of interest.

\section{References}

1. Heusch G. Myocardial ischaemia-reperfusion injury and cardioprotection in perspective. Nat Rev Cardiol. 2020; 17: 773-89.

2. Davidson SM, Ferdinandy P, Andreadou I, Bøtker HE, Heusch G, Ibáñez B, et al. Multitarget Strategies to Reduce Myocardial Ischemia/Reperfusion Injury: JACC Review Topic of the Week. Journal of the American College of Cardiology. 2019; 73: 89-99.

3. Hirschhäuser C, Lissoni A, Görge PM, Lampe PD, Heger J, Schlüter KD, et al. Connexin 43 phosphorylation by casein kinase 1 is essential for the cardioprotection by ischemic preconditioning. Basic research in cardiology. 2021; 116: 21.

4. Hong DM, Lee EH, Kim HJ, Min JJ, Chin JH, Choi DK, et al. Does remote ischaemic preconditioning with postconditioning improve clinical outcomes of patients undergoing cardiac surgery? Remote Ischaemic Preconditioning with Postconditioning Outcome Trial. European heart journal. 2014; 35: 176-83.

5. Wei X, Wu B, Zhao J, Zeng Z, Xuan W, Cao S, et al. Myocardial Hypertrophic Preconditioning Attenuates Cardiomyocyte Hypertrophy and Slows Progression to Heart Failure Through Upregulation of S100A8/A9. Circulation. 2015; 131: 1506-17; discussion 17.

6. Zarbock A, Schmidt C, Van Aken H, Wempe C, Martens S, Zahn PK, et al. Effect of remote ischemic preconditioning on kidney injury among high-risk patients undergoing cardiac surgery: a randomized clinical trial. Jama. 2015; 313: 2133-41.

7. Chen $\mathrm{CH}$, Ferreira JC, Gross ER, Mochly-Rosen D. Targeting aldehyde dehydrogenase 2: new therapeutic opportunities. Physiological reviews. 2014; 94: 1-34. 
8. Gross ER, Zambelli VO, Small BA, Ferreira JC, Chen CH, Mochly-Rosen D. A personalized medicine approach for Asian Americans with the aldehyde dehydrogenase $2 * 2$ variant. Annual review of pharmacology and toxicology. 2015; 55: 107-27.

9. Ma LL, Ding ZW, Yin PP, Wu J, Hu K, Sun AJ, et al. Hypertrophic preconditioning cardioprotection after myocardial ischaemia/reperfusion injury involves ALDH2-dependent metabolism modulation. Redox biology. 2021; 43: 101960.

10. Chen $\mathrm{CH}$, Budas GR, Churchill EN, Disatnik MH, Hurley TD, Mochly-Rosen D. Activation of aldehyde dehydrogenase-2 reduces ischemic damage to the heart. Science. 2008; 321: 1493-5.

11. Ueta CB, Campos JC, Albuquerque RPE, Lima VM, Disatnik MH, Sanchez AB, et al. Cardioprotection induced by a brief exposure to acetaldehyde: role of aldehyde dehydrogenase 2. Cardiovascular research. 2018; 114: 1006-15.

12. Contractor H, Støttrup NB, Cunnington C, Manlhiot C, Diesch J, Ormerod JO, et al. Aldehyde dehydrogenase-2 inhibition blocks remote preconditioning in experimental and human models. Basic research in cardiology. 2013; 108: 343.

13. Pickard JM, Bøtker HE, Crimi G, Davidson B, Davidson SM, Dutka D, et al. Remote ischemic conditioning: from experimental observation to clinical application: report from the 8th Biennial Hatter Cardiovascular Institute Workshop. Basic research in cardiology. 2015; 110: 453.

14. Kitagawa K, Kawamoto T, Kunugita N, Tsukiyama T, Okamoto K, Yoshida A, et al. Aldehyde dehydrogenase (ALDH) 2 associates with oxidation of methoxyacetaldehyde; in vitro analysis with liver subcellular fraction derived from human and Aldh2 gene targeting mouse. FEBS letters. 2000; 476: 306-11.

15. Sun X, Gao R, Li W, Zhao Y, Yang H, Chen H, et al. Alda-1 treatment promotes the therapeutic effect of mitochondrial transplantation for myocardial ischemia-reperfusion injury. Bioactive materials. 2021; 6: 2058-69.

16. Gao RF, Li X, Xiang HY, Yang H, Lv CY, Sun XL, et al. The covalent NLRP3-inflammasome inhibitor Oridonin relieves myocardial infarction induced myocardial fibrosis and cardiac remodeling in mice. Int Immunopharmacol. 2021; 90: 107133.

17. Ackers-Johnson M, Li PY, Holmes AP, O'Brien SM, Pavlovic D, Foo RS. A Simplified, Langendorff-Free Method for Concomitant Isolation of Viable Cardiac Myocytes and Nonmyocytes From the Adult Mouse Heart. Circulation research. 2016; 119: 909-20.

18. Si Y, Bao H, Han L, Chen L, Zeng L, Jing L, et al. Dexmedetomidine attenuation of renal ischaemiareperfusion injury requires sirtuin 3 activation. British journal of anaesthesia. 2018; 121: 1260-71.

19. Wang Z, Sun R, Wang G, Chen Z, Li Y, Zhao Y, et al. SIRT3-mediated deacetylation of PRDX3 alleviates mitochondrial oxidative damage and apoptosis induced by intestinal ischemia/reperfusion injury. Redox biology. 2020; 28: 101343.

20. Lu Z, Bourdi M, Li JH, Aponte AM, Chen Y, Lombard DB, et al. SIRT3-dependent deacetylation exacerbates acetaminophen hepatotoxicity. EMBO Rep. 2011; 12: 840-6. 
21. Amanakis G, Kleinbongard P, Heusch G, Skyschally A. Attenuation of ST-segment elevation after ischemic conditioning maneuvers reflects cardioprotection online. Basic research in cardiology. 2019; 114: 22.

22. Stiermaier T, Jensen JO, Rommel KP, de Waha-Thiele S, Fuernau G, Desch S, et al. Combined Intrahospital Remote Ischemic Perconditioning and Postconditioning Improves Clinical Outcome in ST-Elevation Myocardial Infarction. Circulation research. 2019; 124: 1482-91.

23. Vaibhav K, Braun M, Khan MB, Fatima S, Saad N, Shankar A, et al. Remote ischemic postconditioning promotes hematoma resolution via AMPK-dependent immune regulation. The Journal of experimental medicine. 2018; 215: 2636-54.

24. Ajoolabady A, Aslkhodapasandhokmabad H, Aghanejad A, Zhang Y, Ren J. Mitophagy Receptors and Mediators: Therapeutic Targets in the Management of Cardiovascular Ageing. Ageing research reviews. 2020; 62: 101129.

25. Santin Y, Fazal L, Sainte-Marie Y, Sicard P, Maggiorani D, Tortosa F, et al. Mitochondrial 4-HNE derived from MAO-A promotes mitoCa(2+) overload in chronic postischemic cardiac remodeling. Cell death and differentiation. 2020; 27: 1907-23.

26. Beretta M, Wölkart G, Schernthaner M, Griesberger M, Neubauer R, Schmidt K, et al. Vascular bioactivation of nitroglycerin is catalyzed by cytosolic aldehyde dehydrogenase-2. Circulation research. 2012; 110: 385-93.

27. Ma H, Guo R, Yu L, Zhang Y, Ren J. Aldehyde dehydrogenase 2 (ALDH2) rescues myocardial ischaemia/reperfusion injury: role of autophagy paradox and toxic aldehyde. European heart journal. 2011; 32: 1025-38.

28. Zhai M, Li B, Duan W, Jing L, Zhang B, Zhang M, et al. Melatonin ameliorates myocardial ischemia reperfusion injury through SIRT3-dependent regulation of oxidative stress and apoptosis. Journal of pineal research. 2017; 63.

29. Harris PS, Gomez JD, Backos DS, Fritz KS. Characterizing Sirtuin 3 Deacetylase Affinity for Aldehyde Dehydrogenase 2. Chemical research in toxicology. 2017; 30: 785-93.

30. Koeppen M, Lee JW, Seo SW, Brodsky KS, Kreth S, Yang IV, et al. Hypoxia-inducible factor 2-alphadependent induction of amphiregulin dampens myocardial ischemia-reperfusion injury. Nature communications. 2018; 9: 816.

31. Zhao Y, Wang B, Zhang J, He D, Zhang Q, Pan C, et al. ALDH2 (Aldehyde Dehydrogenase 2) Protects Against Hypoxia-Induced Pulmonary Hypertension. Arteriosclerosis, thrombosis, and vascular biology. 2019; 39: 2303-19.

32. Laitakari A, Ollonen T, Kietzmann T, Walkinshaw G, Mennerich D, Izzi V, et al. Systemic inactivation of hypoxia-inducible factor prolyl 4-hydroxylase 2 in mice protects from alcohol-induced fatty liver disease. Redox biology. 2019; 22: 101145.

33. Ji W, Wei S, Hao P, Xing J, Yuan Q, Wang J, et al. Aldehyde Dehydrogenase 2 Has Cardioprotective Effects on Myocardial Ischaemia/Reperfusion Injury via Suppressing Mitophagy. Frontiers in pharmacology. 2016; 7: 101. 
34. Kornfeld OS, Hwang S, Disatnik MH, Chen CH, Qvit N, Mochly-Rosen D. Mitochondrial reactive oxygen species at the heart of the matter: new therapeutic approaches for cardiovascular diseases. Circulation research. 2015; 116: 1783-99.

35. Guéraud F. 4-Hydroxynonenal metabolites and adducts in pre-carcinogenic conditions and cancer. Free radical biology \& medicine. 2017; 111: 196-208.

36. Mak S, Lehotay DC, Yazdanpanah M, Azevedo ER, Liu PP, Newton GE. Unsaturated aldehydes including 4-OH-nonenal are elevated in patients with congestive heart failure. Journal of cardiac failure. 2000; 6: 108-14.

37. Santin Y, Fazal L, Sainte-Marie Y, Sicard P, Maggiorani D, Tortosa F, et al. Mitochondrial 4-HNE derived from MAO-A promotes mitoCa(2+) overload in chronic postischemic cardiac remodeling. Cell Death Differ. 2020; 27: 1907-23.

38. Eng MY, Luczak SE, Wall TL. ALDH2, ADH1B, and ADH1C genotypes in Asians: a literature review. Alcohol research \& health : the journal of the National Institute on Alcohol Abuse and Alcoholism. 2007; 30: 22-7.

39. Yukawa Y, Muto M, Hori K, Nagayoshi H, Yokoyama A, Chiba T, et al. Combination of $A D H 1 B * 2 / A L D H 2 * 2$ polymorphisms alters acetaldehyde-derived DNA damage in the blood of Japanese alcoholics. Cancer science. 2012; 103: 1651-5.

40. Li H, Borinskaya S, Yoshimura K, Kal'ina N, Marusin A, Stepanov VA, et al. Refined geographic distribution of the oriental ALDH2*504Lys (nee 487Lys) variant. Annals of human genetics. 2009; 73 : $335-45$.

\section{Figures}




\section{Figure 1}

A



D

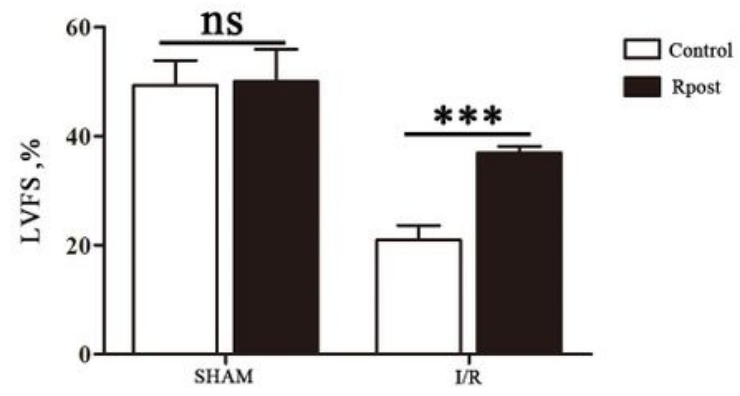

$\mathrm{F}$



B

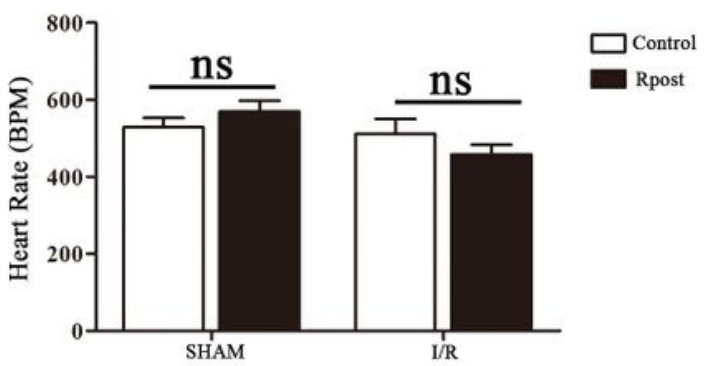

$\mathrm{C}$



$\mathrm{E}$

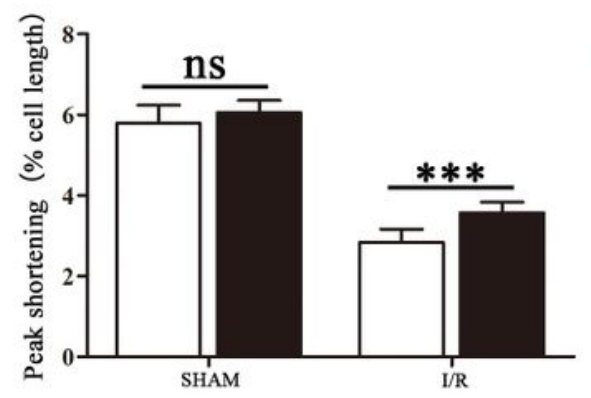

Figure 1

Rpost treatment preserves cardiac function after $\mathrm{I} / \mathrm{R}$.

Representative images of echocardiography tracing in l/R mice treated with Rpost in each group after 24 h reperfusion (A). Heart rate (BPM; B). Left ventricular ejection fraction (LVEF; C). Left ventricular fractional shortening (LVFS; D). Peak shortening (\% cell lengthening; E). Maximal shortening velocity $(\mathrm{dl} / \mathrm{dt} ; \mathrm{F})$. Data are depicted as the mean \pm SEM. Statistical significance was determined by two-way 
ANOVA with a post-hoc Holm-Sidak test, ns, not significant; ${ }^{*} \mathrm{P}<0.05$; ${ }^{* \star *} \mathrm{P}<0.001$; compared with the control group.

\section{Figure 2}

A

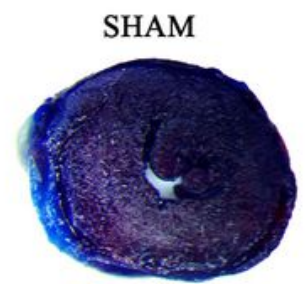

B
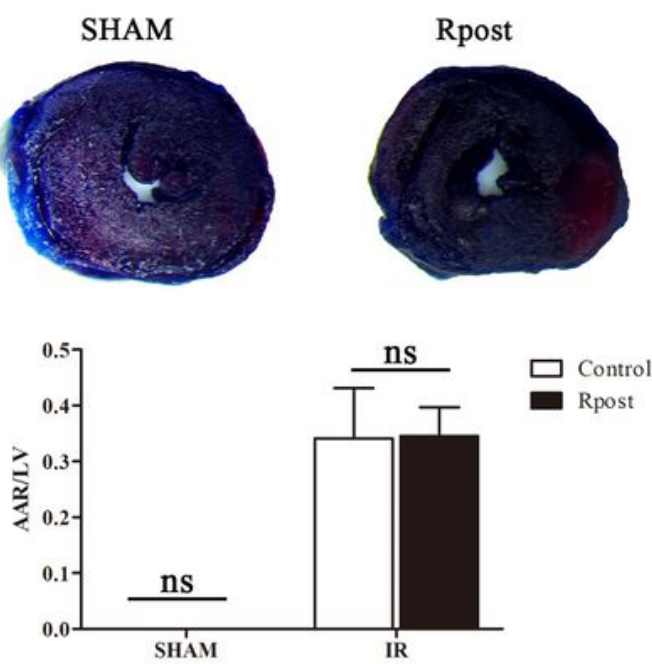

C

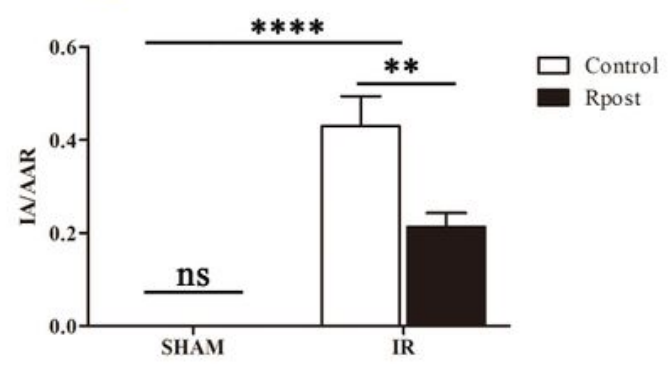

D

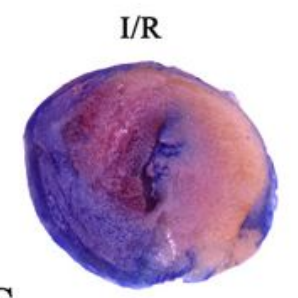

E

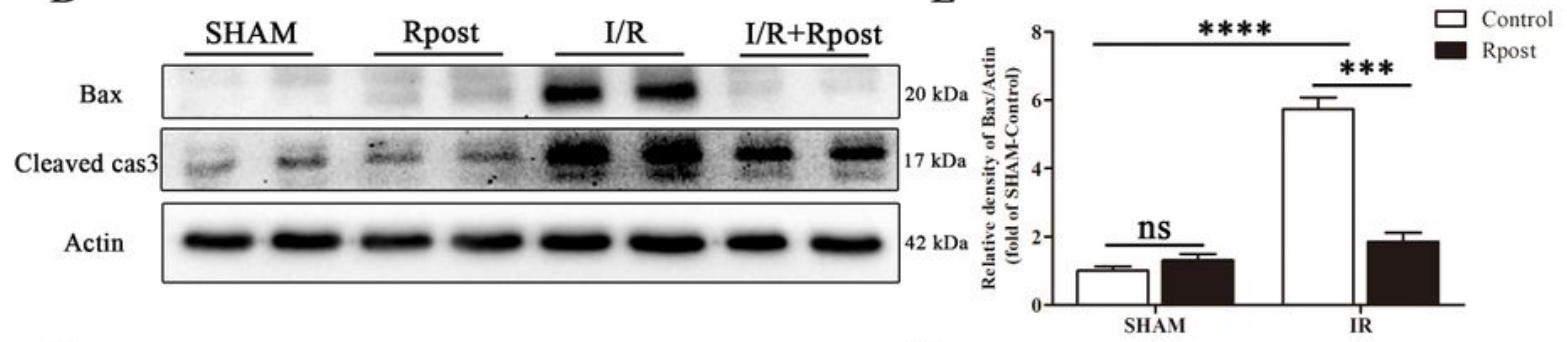

F

DAPI
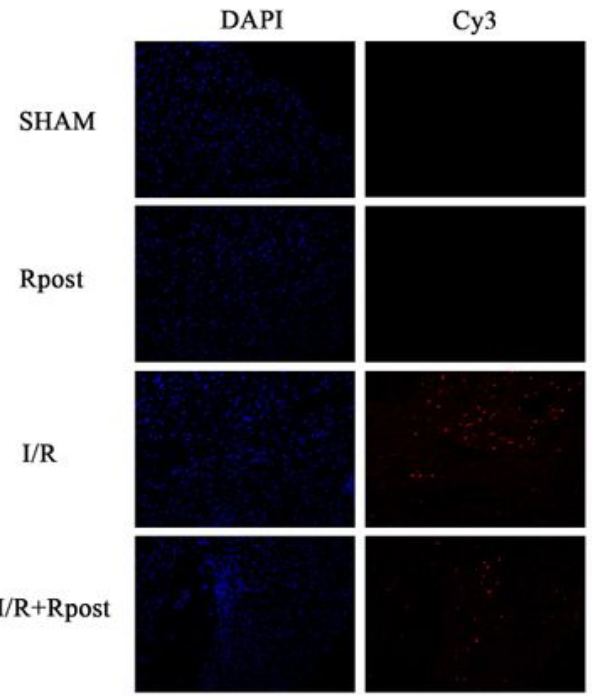

Merge


G

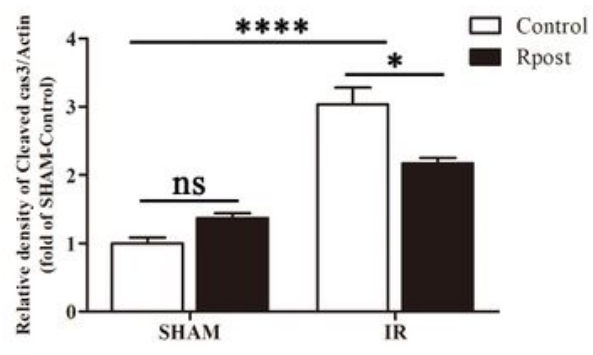

$\mathrm{H}$



Figure 2

Rpost treatment ameliorates $\mathrm{I} / \mathrm{R}$ injury in mice. 
Representative images of Evans blue dye and TTC staining (A). Ratio of risk area to left ventricular area in each group (AAR/LV; B). Change in infarction size induced by Rpost or without Rpost (IA/AAR; C).

Western blot of cleaved-caspase $3(D, G)$ and Bax (D, E) levels in cardiomyocytes after I/R treatment and Rpost treatment. Fluorescence imaging of cardiomyocyte apoptosis induced by I/R treatment with Rpost. The cell nuclei were stained with DAPI (blue). Red represents apoptotic cardiomyocytes (F and H). Data are depicted as the mean \pm SEM. Statistical significance was determined by two-way ANOVA with a posthoc Holm-Sidak test, ns, not significant; ${ }^{2} P<0.05 ; * \star P<0.001 ; * \star \star P<<0.001 ; * \star \star * P<0.0001$.

\section{Figure 3}

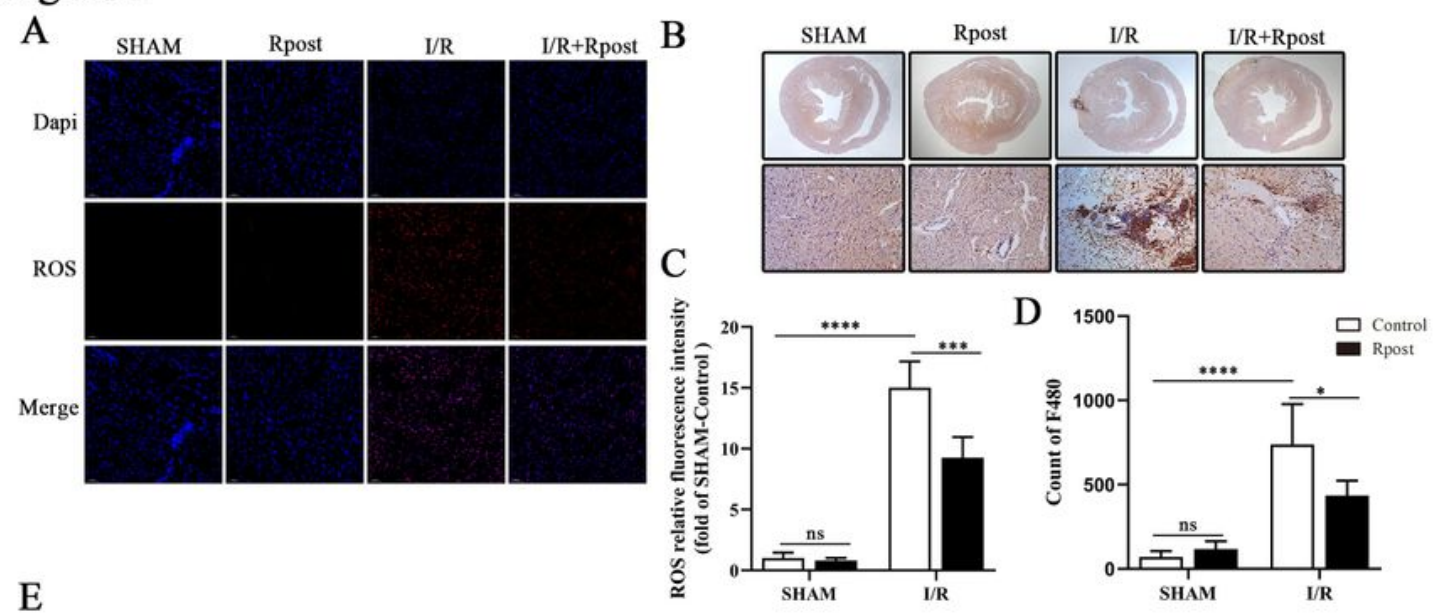

$\mathrm{E}$ $\underline{\text { SHAM }}$ Rpost $\quad$ I/R $\quad$ //R+Rpost

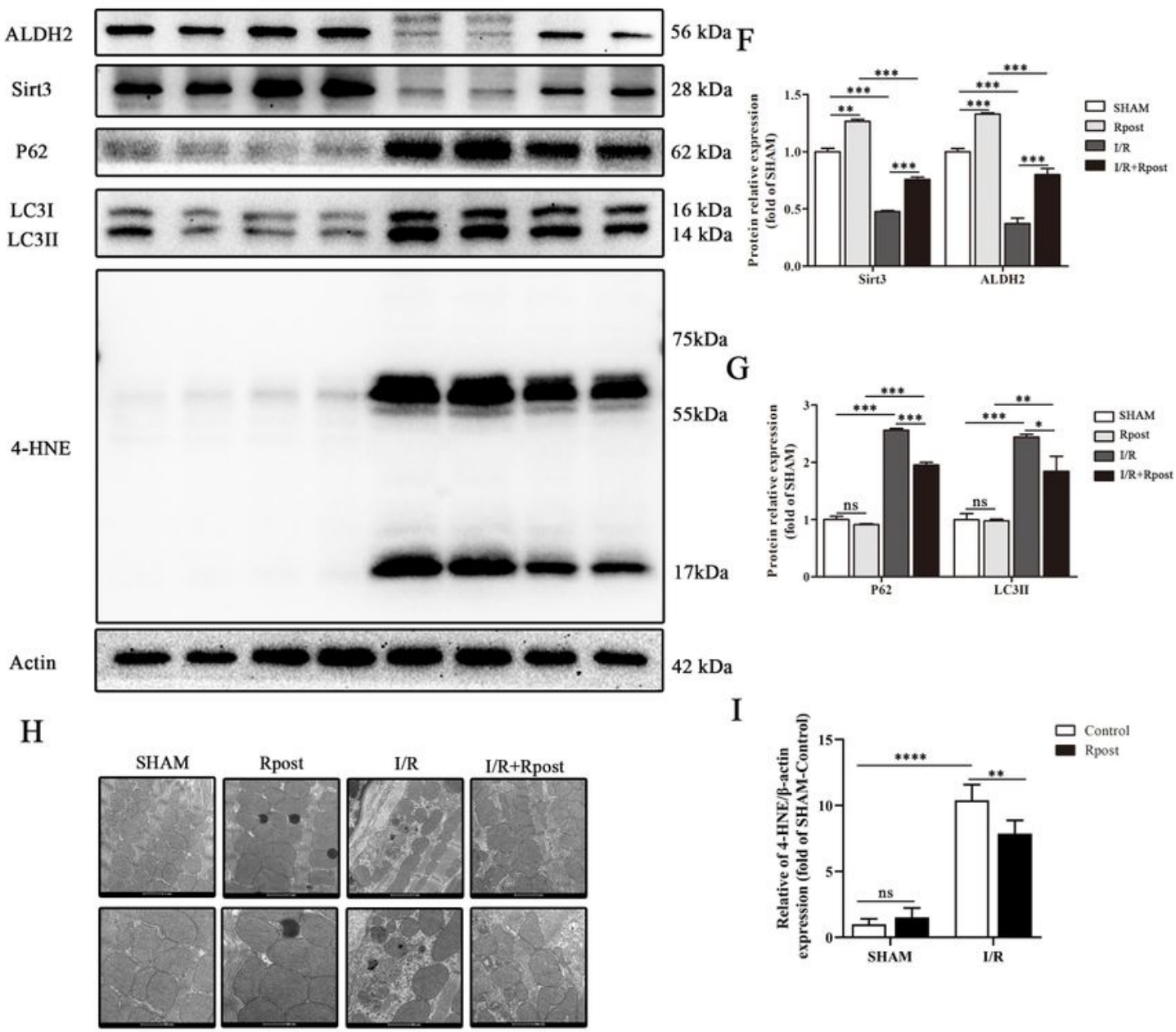




\section{Figure 3}

Rpost protects myocardium from I/R injury through ALDH2/SIRT3 regulation of autophagy and reduction of 4-HNE levels in mice

Representative images of DHE-stained heart sections from mice $1 \mathrm{~d}$ after l/R. Scale bar $=50 \mu \mathrm{m}$. (A). Relative index of ROS fluorescence (C). Representation of myocardial macrophage infiltration (F480, B) and related statistics (D). Representative western blot of ALDH2, SIRT3, P62, LC3II, and 4-HNE levels in mice treated with Rpost and control animals (E). Effects of Rpost on ALDH2 (F), SIRT3 (F), P62 (G), LC3II (G), and 4-HNE (I) expression in I/R mouse model. Transmission electron microscopy (TEM) images of the left ventricle $(H)$. Data are depicted as the mean \pm SEM. Statistical significance was determined by two-way ANOVA with a post-hoc Holm-Sidak test, ${ }^{*} P<0.05$, ${ }^{\star *} \mathrm{P}<0.001,{ }^{\star \star \star} \mathrm{P}<0.001,{ }^{\star \star \star \star *} \mathrm{P}<0.0001$. 
Figure 4

A

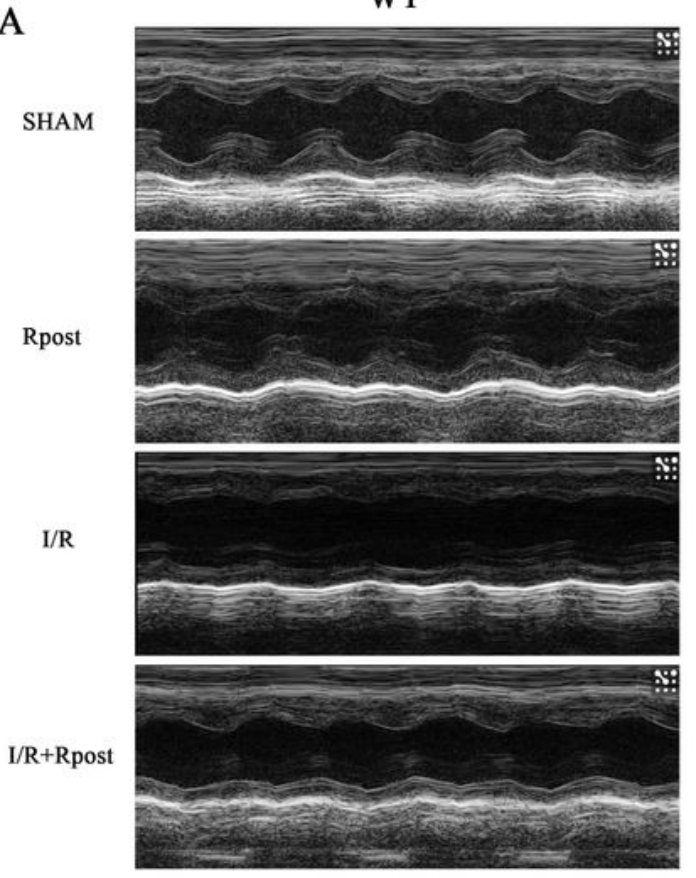

B

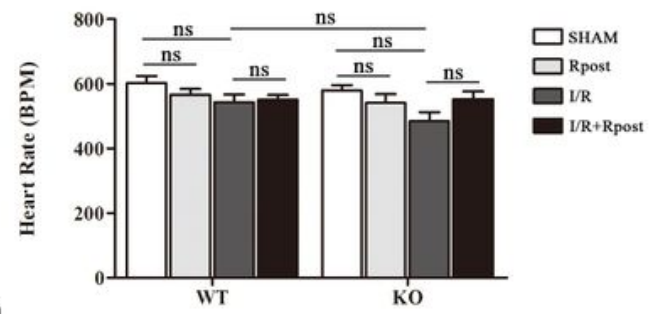

D

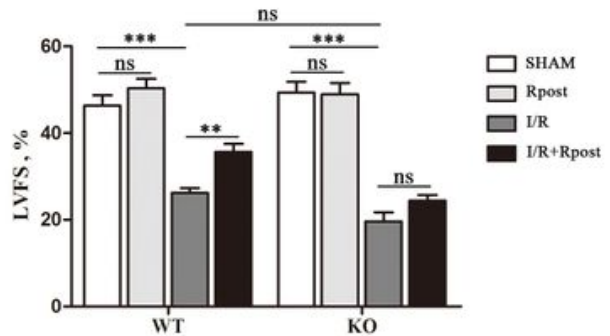

$\mathrm{F}$

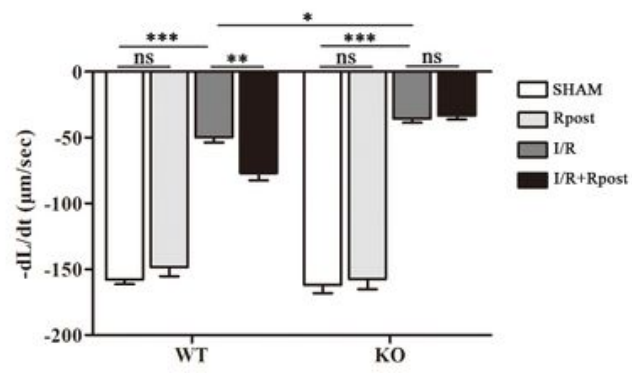

$\mathrm{C}$
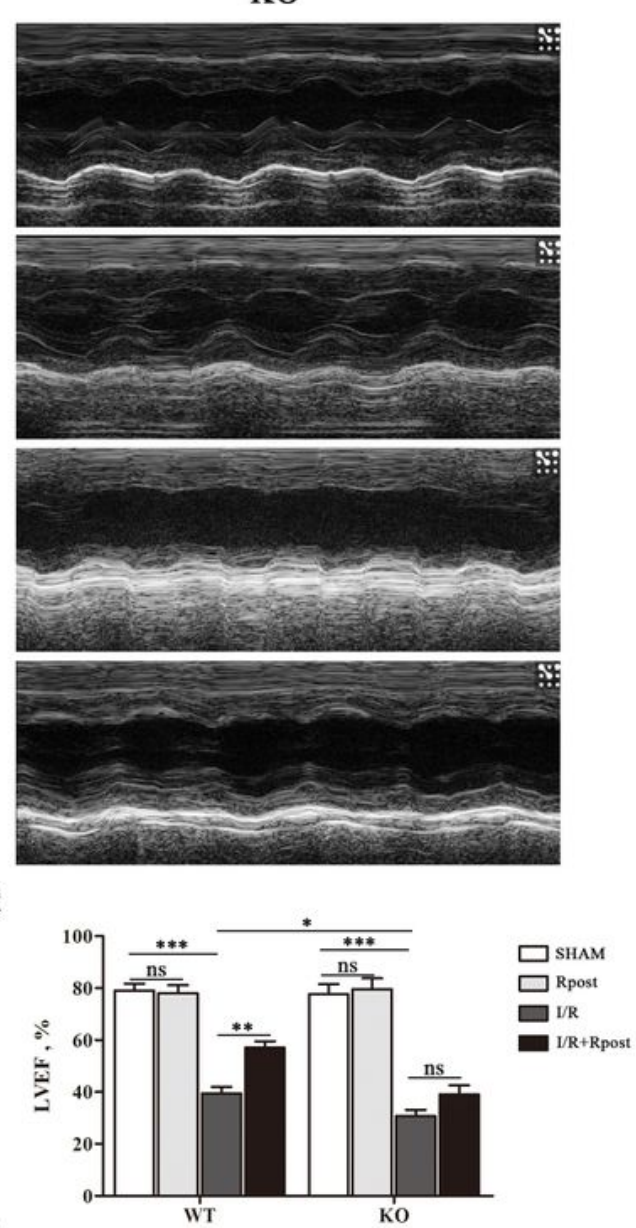

$\mathrm{E}$



Figure 4

\section{ALDH2 deficiency blocks protective effect of Rpost on I/R injury in mice}

Representative images of echocardiography tracing in I/R mice treated with Rpost in the WT and ALDH2$\mathrm{KO}$ groups after $24 \mathrm{~h}$ of reperfusion (A). Heart rate (BPM; B). Left ventricular ejection fraction (LVEF; C). Left ventricular fractional shortening (LVFS; D). Peak shortening (\% cell lengthening; E). Maximal 
shortening velocity ( $\mathrm{dl} / \mathrm{dt} ; \mathrm{F})$. Data are depicted as the mean $\pm \mathrm{SEM}$. Statistical significance was determined by two-way ANOVA with a post-hoc Holm-Sidak test, ns, not significant; ${ }^{*}<<0.05 ;{ }^{*} \mathrm{P}<0.001$; $* * * P<0.001$.

\section{Figure 5}

A
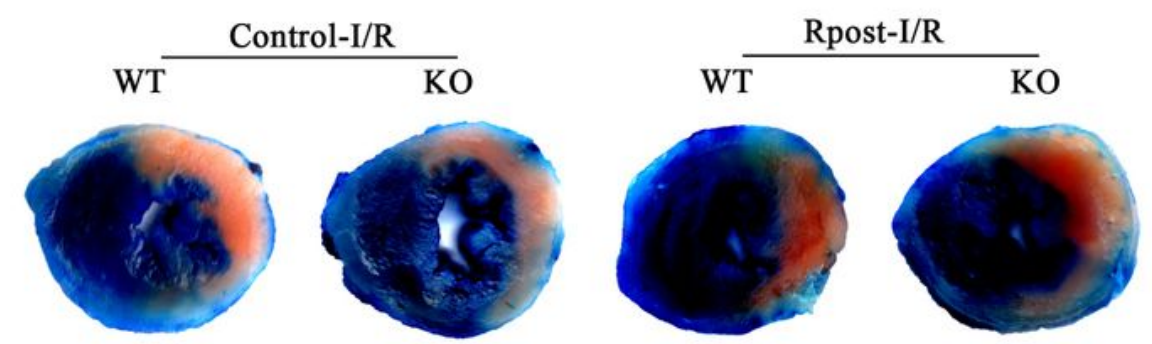

B

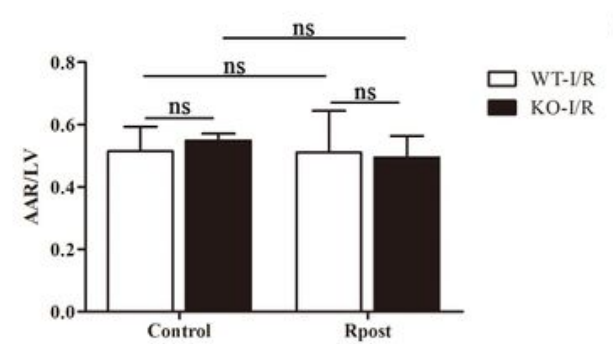

$\mathrm{C}$



D
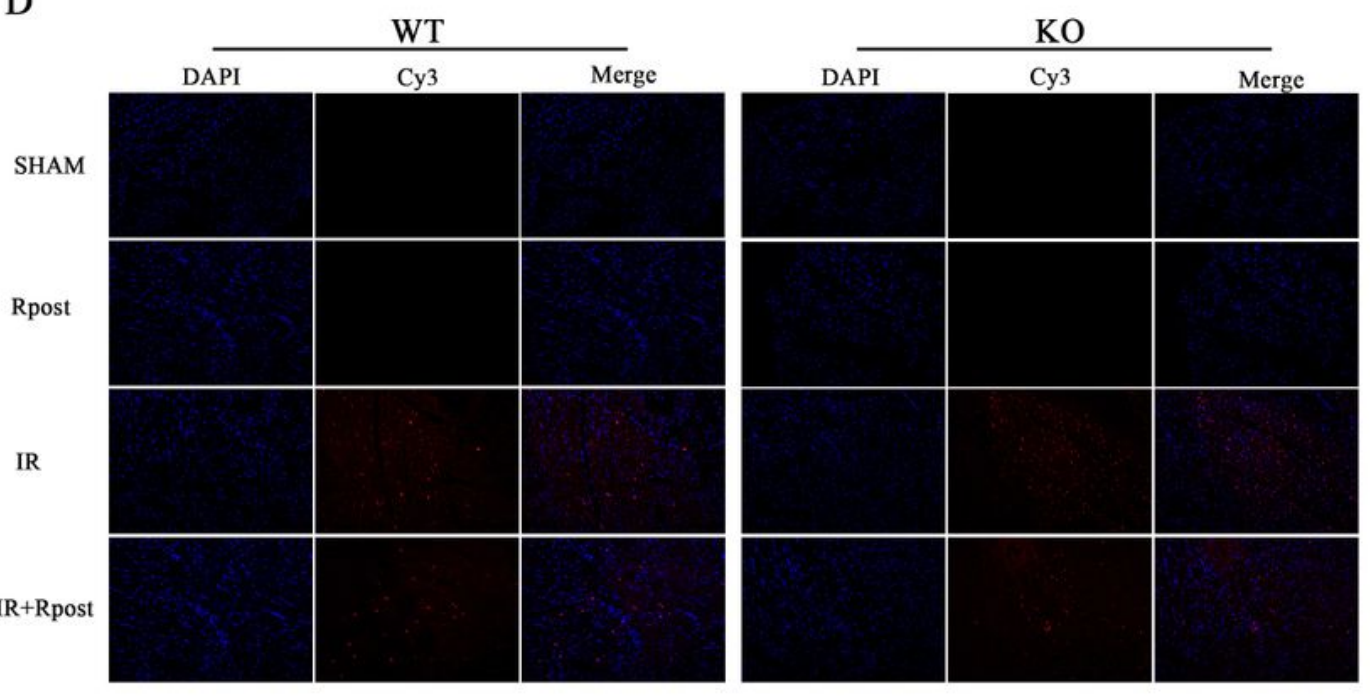

$\mathrm{E}$

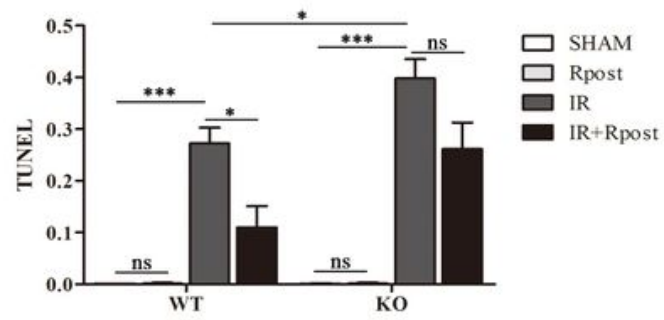

Figure 5 
Representative images of Evans blue dye and TTC staining (A). Ratio of risk area to left ventricular area in each group (AAR/LV; B). Change in infarction size induced by Rpost or without Rpost in the WT and KO groups (IA/AAR; C). Fluorescence imaging of cardiomyocyte apoptosis induced by I/R treatment with Rpost. Cell nuclei were stained with DAPI (blue). Red represents apoptotic cardiomyocytes (D and E). Data are depicted as the mean \pm SEM. Statistical significance was determined by two-way ANOVA with a post-hoc Holm-Sidak test, ns, not significant; ${ }^{*} P<0.05 ; * \star P<0.001 ; * \star * P<0.001$.

\section{Figure 6}
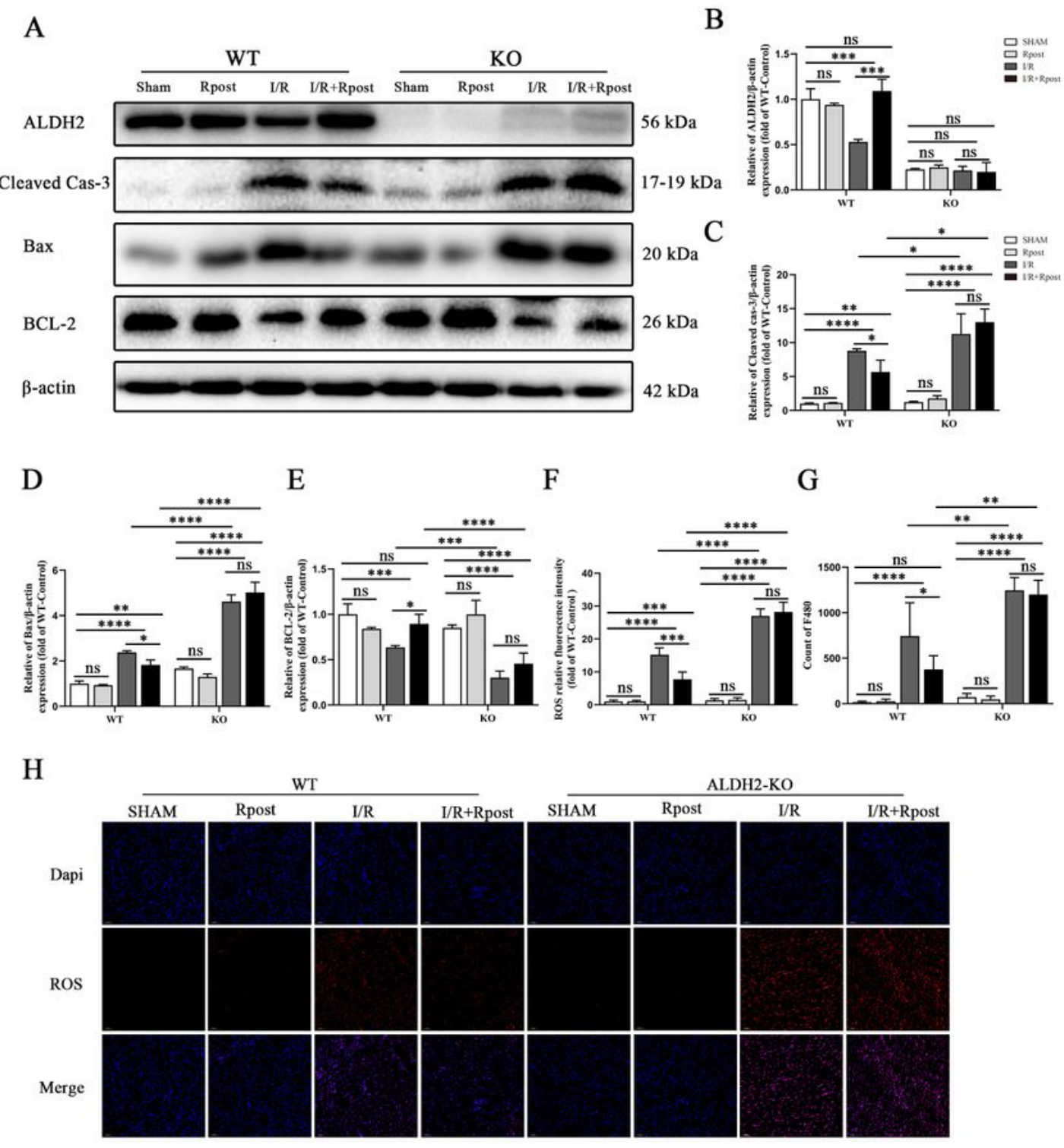

I

\begin{tabular}{|c|c|c|c|c|c|c|c|}
\hline \multicolumn{4}{|c|}{ WT } & \multirow{2}{*}{\multicolumn{4}{|c|}{ ALDH2-KO }} \\
\hline SHAM & Rpost & I/R & IR+RposI & SHAM & Rpost & & $1 / R+R p o s t$ \\
\hline$x$ & & 3 & & I) & $(x)$ & (3) & 3 \\
\hline & & & & & & & \\
\hline
\end{tabular}


Figure 6

\section{Rpost reduces ROS damage and inflammatory infiltration after I/R was blocked by ALDH2}

Representative western blot of ALDH2, cleaved caspase-3, Bax, and BCL-2 in mice treated with Rpost and control WT and ALDH2-KO groups (A). After I/R, Rpost downregulated cleaved caspase-3 (C) and Bax (D) expression and upregulated ALDH2 (B) and Bcl-2 (E) expression, but ALDH2 blocked this effect.

Representative images of DHE-stained heart sections from mice $1 \mathrm{~d}$ after $\mathrm{I} / \mathrm{R}(\mathrm{H})$. Relative index of ROS fluorescence $(F)$. Representation of myocardial macrophage infiltration (F480, I) and related statistics $(\mathrm{G})$. Data are depicted as the mean \pm SEM. Statistical significance was determined by two-way ANOVA with a post-hoc Holm-Sidak test, ns, not significant; ${ }^{*} P<0.05 ; * \star P<0.001 ; * \star \star P<0.001, * \star \star \star P<0.0001$. 
Figure 7



B

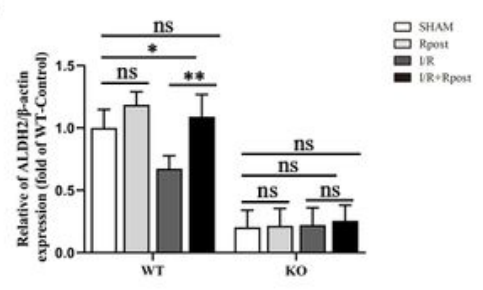

C



E
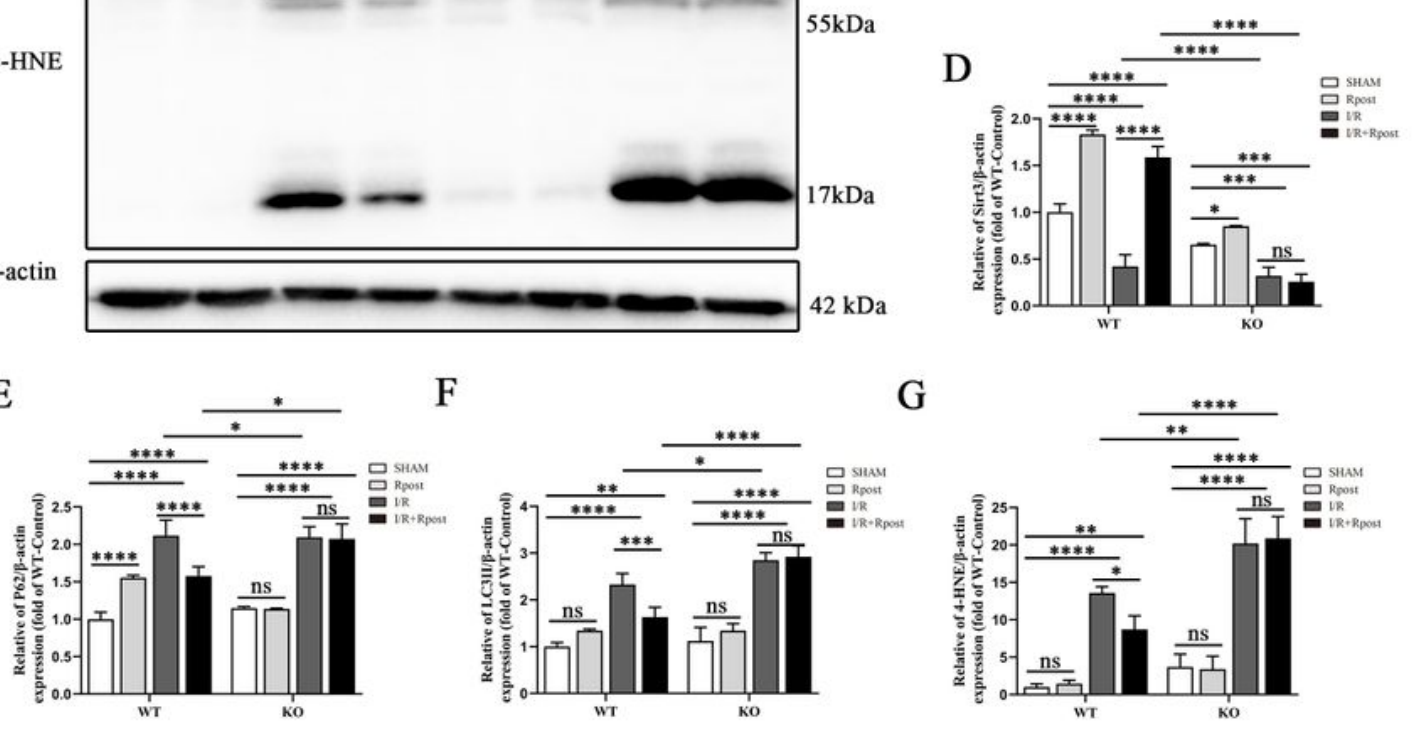

$\mathrm{H}$

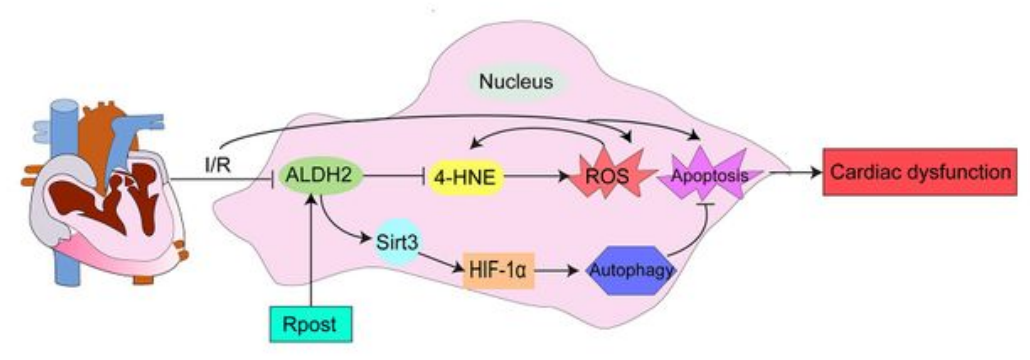

Figure 7

Rpost exhibits cardio-protection after MI/RI by eliminating 4-HNE and regulating autophagy through ALDH2/SIRT3/HIF1a signaling pathway

Representative western blot of ALDH2, SIRT3, HIF1a, P62, LC3II, and 4-HNE expression (A). In the I/R model, Rpost-treatment of the WT group upregulated the expression of ALDH2 (B), SIRT3 (C), and HIF1a 
(D) and regulated autophagy-related proteins, P62 and LC3II, (E, F). However, after ALDH2-KO, the effect of Rpost was blocked. Furthermore, Rpost reduced 4-HNE levels, but there was no significant difference in the ALDH2-KO group (G). Rpost cardio-protection after MI/RI by eliminating 4-HNE and regulating autophagy through the ALDH2/SIRT3/HIF1a signaling pathway $(\mathrm{H})$. Data are depicted as the mean \pm SEM. Statistical significance was determined by two-way ANOVA with a post-hoc Holm-Sidak test, ns, not significant; ${ }^{*} P<0.05 ; * \star P<0.001 ; * \star * P<0.001 ; * \star * P<0.001$. 